\title{
Subnanometer-resolved measurement of the tunneling effective mass using bulk plasmons
}

\author{
V. Stolojan \\ Advanced Technology Institute, University of Surrey, Guildford GU2 7XH, England \\ P. Moreau \\ Institut des Matériaux Jean Rouxel, Université de Nantes-CNRS, Laboratoire de Chimie des Solides, 44322 \\ Nantes, France \\ M. J. Goringe \\ School of Engineering, University of Surrey, Guildford GU2 7XH, England \\ S. R. P. Silva ${ }^{\text {a) }}$ \\ Advanced Technology Institute, University of Surrey, Guildford GU2 7XH, England
}

(Received 31 August 2005; accepted 23 February 2006; published online 22 March 2006)

\begin{abstract}
Superlattices are periodic structures where the constituents alternate between low- and high-bandgap materials; the resulting quantum confinement tailors the resulting device properties and increases their operating speed. Amorphous carbon is an excellent candidate for both the well and barrier layers of the superlattices, leading to a fast and reliable device manufacturing process. We show theoretically and experimentally that, using low energy-loss spatially resolved spectroscopy, we can characterize the component layers of a superlattice. We measure quantum confinement of the electron wave function in the superlattice's wells and calculate the effective tunneling mass for amorphous carbon superlattices as $m^{*}=0.067 m_{e}$. This effective mass makes diamondlike carbon films as feasible candidate for electronic devices. (C) 2006 American Institute of Physics. [DOI: 10.1063/1.2188593]
\end{abstract}

Diamond-like carbon films have optical band gaps from $1.2-4.0 \mathrm{eV}$ controllable through the deposition parameters, such as the plasma power. ${ }^{1}$ They are an attractive option for the semiconductor industry due to the inexpensive, fast and reliable manufacturing method. When the deposition parameter is varied cyclically during deposition, we obtain a bandgap modulation in an essentially homogenous material system, ${ }^{2,3}$ leading to higher device speeds and controllable electronic properties. The confining potential of a band-gapmodulated artificial structure on the electron wave function leads to the quantization of the particle momentum and energy, controlled by the well's width and depth and the number of alternating barrier and well layers. ${ }^{4,5}$ This can lead to the tailoring of devices for specific applications, such as frequency generators for the mobile phone industry. The design and testing of manufacturing processes for superlattices require not only the ability to characterize the morphology of the individual layers, but also their individual electronic properties. This places electron energy loss spectroscopy (EELS) in a transmission electron microscope (TEM) in the unique position of providing all this information. Here, we use an alternative method of acquiring spectral information on a subnanometer scale, and then set the theoretical basis for interpreting the measured collective excitation on the subnanometer scale. We then extract the tunneling effective mass by modeling the changes measured in the collective excitations using the "particle-in-a-box" quantum confinement model.

In an electron microscope, it is possible to image the barrier and well layers constituent of a superlattice, even

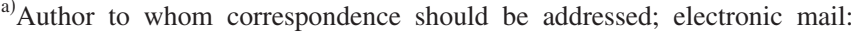
s.silva@surrey.ac.uk
}

when their respective physical properties are very similar, at large defoci [Fig. 1(a)]. ${ }^{6}$ Energy loss spectroscopic profiling provides spatially resolved energy-loss spectra across linear features, under parallel illumination. ${ }^{7}$ This method utilizes the manner in which a Gatan imaging filter forms images and energy-loss spectra, by collecting two-dimensional data sets with one axis, the energy loss and the other axis the spatial dimension normal to the linear feature of interest [Fig. 1(b)].

Because the spatial dimension is acquired in parallel with the energy loss information, this method offers a very accurate positioning of each energy loss spectrum with respect to the linear feature of interest.

The superlattices were deposited on (001)Si substrates using plasma enhanced chemical vapor deposition, with the band-gap modulated by alternating the dc self-bias between -190 and $-265 \mathrm{~V}$, under computer control. ${ }^{2,3}$ The nominal dimensions of the sample are contained in its name, where B140W80 signifies a barrier width of $140 \AA$ and a well width of $80 \AA$, respectively. Cross-sectional samples for TEM were prepared by mechanical thinning and polishing, followed by ion beam polishing. Test barrier and well films were also deposited, under identical conditions as the barrier and well layers within a superlattice; these were removed from the Si substrate by using a $\mathrm{HF}: \mathrm{HNO}_{3}$ solution and then floated onto $\mathrm{Cu}$ TEM grids.

For each of the superlattices and as-deposited tunnel and barrier samples, we collected images similar to Fig. 1(b). The typical energy dispersion was $\sim 0.1 \mathrm{eV} /$ pixel while the typical dispersion of the spatial dimension was $\sim 0.4 \mathrm{~nm}$. The origin of the energy loss scale was defined to within $20 \mathrm{meV}$, while the energy dispersion was determined to within $5 \mathrm{meV}$. A modified Lorentzian curve was used to fit the plasmon energy to within $10 \mathrm{meV}$. 

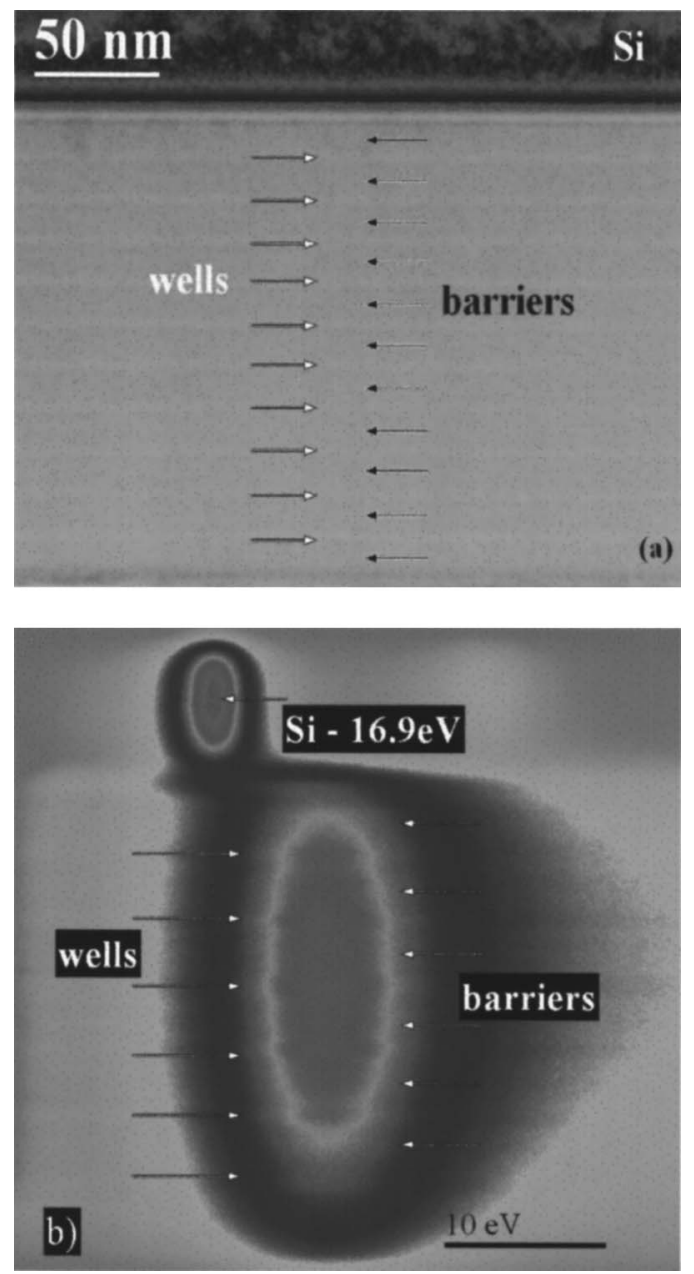

FIG. 1. (a) Defocused $(-57 \mu \mathrm{m})$ energy-filtered image of the superlattice B120W40, with the barriers and wells indicated by the arrows. (b) The corresponding ELSP image to (a), at the same defocus for visibility, showing the plasmon energy across the superlattice (the zero loss peak has been subtracted). The vertical dimension is identical to that in (a), to within a scaling factor. Subsequent experiments were performed at gaussian focus to eliminate elastic contributions to the plasmon peak energy.

Figure 2 shows the variation of the plasmon energy across two of the superlattices, B140W80 and B120W40; note that the energy difference of the plasmon peaks in the barrier and well decreases for the narrower well.

The short acquisition times used $(0.5 \mathrm{~s})$ minimize the loss of spatial resolution due to drift (broadening $\sim 0.1$ pixels) while the small energy range studied $(0-25 \mathrm{eV})$ means

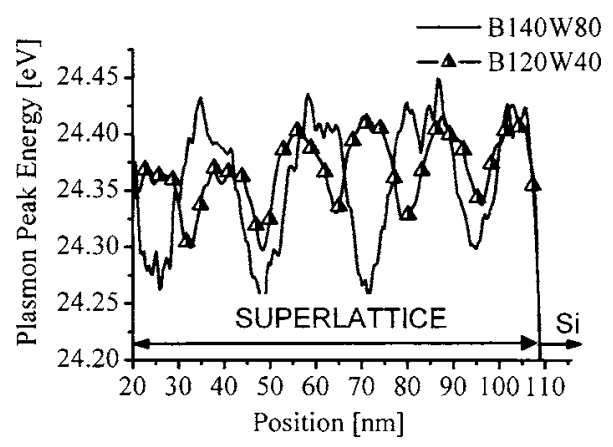

FIG. 2. Comparison of the plasmon energy profiles across superlattices B140W80 and B120W40, showing a decrease in the barrier-well plasmon peak energy difference with decreasing well width, suggesting an increase in

the well band gap.
Downloaded 30 Mar 2009 to 131.227.178.132. Redistribution subject to AlP license or copyright; see http://apl.aip.org/apl/copyright.jsp

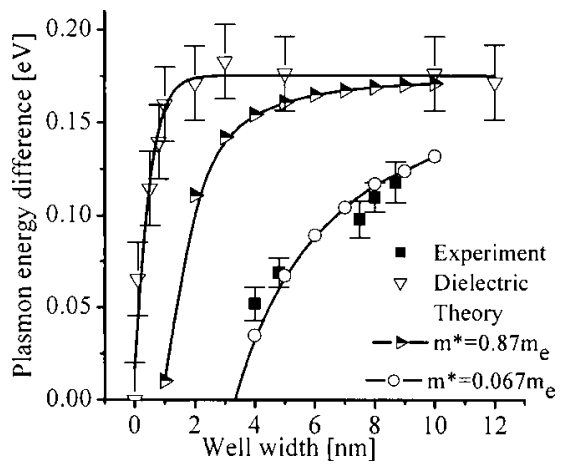

FIG. 3. Comparison of the barrier-well plasmon peak difference calculated with the effective mass as a parameter (half-filled triangles $m^{*}=0.87 m_{e}$ and open circles $m^{*}=0.067 m_{e}$ ). The experimental results (filled squares) fit the lower effective mass. Also shown is the calculated contribution from the adjacent bulk plasmons and interface plasmons (open triangles), which is negligible for the "sandwiched" layer thickness down to $1 \mathrm{~nm}$ and accountable for layer widths down to $0.5 \mathrm{~nm}$.

we can also neglect the effect of chromatic aberration. The resulting instrumental spatial resolution is approximately $0.7 \mathrm{~nm}$ (for a dispersion of $\sim 0.45 \mathrm{~nm} /$ pixel).

Bulk collective modes of oscillation extend (normal to the beam direction) typically over $5-10 \mathrm{~nm} .^{8}$ However, an interface between two media leads to the establishment of a surface mode of oscillation, which acts to screen out the bulk plasmons from each other. This is known as the "begrenzungs" effect ${ }^{8,9}$ which manifests as the replacement (not overlap) of the bulk plasmon by the interface plasmon, leading to an effective screening. In order to evaluate the increase in plasmon energy in the middle of the well caused by the contributions from the interface and barrier plasmons, we simulate the dielectric response for a theoretical onedimensional well of variable width, confined by infinitely long barriers. We use the classical dielectric approach ${ }^{10}$ to calculate the energy loss of the relativistic electron, as a function of its position across the well, for a sandwich interface. ${ }^{11}$ Dielectric functions derived from the low energyloss spectra of the well and barrier test layers were input in a dedicated program. ${ }^{12}$

Before comparing with the experimental results, the calculated positions of the plasmon energies across a well are convoluted with our estimated spatial resolution $(\sim 0.7 \mathrm{~nm})$.

While the bulk plasmons are delocalized events, which can extend over several nanometers, the presence of interface excitations and their effective screening allows for the measurement of bulk plasmons within layers narrower than their extinction distances. Only when the well width becomes $<1 \mathrm{~nm}$, there is a more significant contribution to the plasmon energy in the well from the surface and barrier plasmons (Fig. 3-open triangles). Even for well widths down to $0.5 \mathrm{~nm}$, it is still possible to interpret the bulk plasmon energy by modeling the changes and applying a suitable correction.

Figure 3 (squares) also compares the experimental difference between the barrier and well plasmon energies, as a function of well width. The change in plasmon energy $E_{P}$ can be modeled using the nearly free-electron model, with each electron bound by the gap energy: ${ }^{13}$ 


$$
E_{P}^{2}=\hbar^{2} \frac{\rho_{\mathrm{el}} e^{2}}{\epsilon_{0} m}+E_{g}^{2},
$$

where $\rho_{\mathrm{el}}$ and $m$ are the density of valence electrons and their mass, $e$ is the electron charge, $\hbar$ is the reduced Planck constant, and $\epsilon_{0}$ is the permittivity of vacuum. $E_{g}$ is the Penn gap, which adjusts the free electron model at small values of the wave vector $k$ for Bragg reflections and Umklapp processes. $^{14}$

Using the measured ${ }^{2}$ refractive indices $n_{\text {barrier }}=2.1$ and $n_{\mathrm{well}}=2.2$ and the plasmon energies measured on the test barrier and well layers $\left(E_{P \text { barrier }}=24.400 \mathrm{eV}\right.$ and $E_{P \text { well }}$ $=24.225 \mathrm{eV}$ ), we calculate [Eq. (1)] the Penn gap energies to be $E_{g \text { barrier }}=13.210 \mathrm{eV}$ and $E_{g \text { well }}=12.360 \mathrm{eV}$. These Penn gap energies are then used to determine the depth of the one-dimensional box ${ }^{4}$ with which we model the changes in the ground state of the trapped electron wave function (depth $0.425 \mathrm{eV}=$ one-half of the band-gap difference), as a function of well thickness. The theoretical increase in the Penn bandgap energy as a function of the well thickness is now used to relate back to the plasmon energy [Eq. (1)]; however, this requires knowledge of the effective mass for amorphous carbons. The effective mass found experimentally (using EELS) and theoretically ${ }^{15,16}$ for amorphous carbons is $m^{*}=0.87 m_{e}$, while Silva et $a l^{2,3}$ have shown a tunneling effective mass for amorphous carbon superlattices $m^{*}=0.067 m_{e}$. Assuming that the free-electron component in Eq. (1) does not change with changing well width, we obtain the difference between the bulk and the well plasmon energies as a function of well width, for the two effective mass hypotheses (Fig. 3, half triangles, open circles). From Fig. 3, it is clear that $m^{*}$ $=0.067 m_{e}$ is a good fit for our measured changes in the plasmon energies, when compared with $m^{*}=0.87 m_{e}$. If the effective mass is used as a parameter in fitting the experimental data in Fig. 3, then we find that this method could be used to determine the effective mass to within $0.02 m_{e}$. The apparent discrepancy between the effective masses available in literature can be explained as follows: The Penn band gap used in Eq. (1) is modeled using particle-in-a-box calculations, which are based on the matching of the wave function at the boundaries, as constrained by the boundary conditions. Hence, the effective mass resulting from this fitting is related to the boundaries between wells and barriers, i.e., the tunneling effective mass, as opposed to $m^{*}=0.87 m_{e}$, which is related to dipole transitions in EELS and thus, to a bulk value for the layers.
Using spatially resolved low-energy loss spectra acquired in a TEM, under parallel illumination, we have characterized the electronic structure of amorphous carbon superlattices. We have shown that the changes in plasmon energy measured for wells, as a function of decreasing well width, are due to quantum confinement and can be modeled using the particle-in-a-box theory. By modeling the contribution of the interface plasmon excitations, we show that the delocalized bulk collective excitations can be used to characterize the electronic properties of the wells and barriers, respectively, down to well thicknesses of $\sim 0.5 \mathrm{~nm}$, due to the effective screening provided by the interfaces. We find that the tunneling effective mass is $m^{*}=0.067 m_{e}$. We show that this does not contradict the effective mass value found for amorphous carbon, ${ }^{15,16}$ as it refers to different symmetry points in the band diagram. The low effective mass, coupled with the high coherence length of carriers, ${ }^{2,3}$ makes possible high frequency $(>10 \mathrm{GHz})$ large area electronics, as recently shown. ${ }^{17}$

${ }^{1}$ S. R. P. Silva, G. A. J. Amaratunga, and C. P. Constantinou, J. Appl. Phys. 72, 1149 (1992)

${ }^{2}$ S. R. P. Silva, G. A. J. Amaratunga, C. J. Woodburn, M. E. Welland, and S. Haq, Jpn. J. Appl. Phys., Part 1 33, 6458 (1994).

${ }^{3}$ S. R. P. Silva, G. A. J. Amaratunga, Rusli, S. Haq, and E. K. Salje, Thin Solid Films 253, 20 (1994).

${ }^{4}$ F. Mandl, Quantum Mechanics, (Wiley, New York, 1992), p. 30.

${ }^{5}$ D. K. Ferry and S. M. Goodnick, in Transport in Nanostructures, Cambridge Studies in Semiconductor Physics and Microelectronic Engineering, Vol. 6, edited by H. Ahmed, M. Pepper, and A. Broers (Cambridge University Press, Cambridge, UK, 1997), p. 28.

${ }^{6}$ C. A. Davis, S. R. P. Silva, R. E. Dunin-Borkwoski, G. A. J. Amaratunga, K. M. Knowles, and W. M. Stobbs, Phys. Rev. Lett. 75, 4258 (1995).

${ }^{7}$ T. Walther, Ultramicroscopy 96, 401 (2003).

${ }^{8}$ A. Howie, in Topics in Electron Diffraction and Microscopy of Materials, edited by B. Hirsch (Institute of Physics, Bristol, 1999), p. 79.

${ }^{9}$ A. Howie, Ultramicroscopy 11, 141 (1983).

${ }^{10}$ R. Garcia-Molina, A. Gras-Marti, A. Howie, and R. H. Ritchie, J. Phys. C 18, 5345 (1985).

${ }^{11}$ P. Moreau, N. Brun, C. A. Walsh, C. Colliex, and A. Howie, Phys. Rev. B 56, 6774 (1997).

${ }^{12} \mathrm{C}$. A. Walsh, Computer Programmes for the Calculation of Electron Energy-Loss Spectra from Interfaces Between Dielectric Media (Cavendish Laboratory, Cambridge, UK, 1992).

${ }^{13}$ R. F. Egerton, Electron Energy Loss Spectroscopy in the Electron Microscope, 2nd ed. (Plenum, New York, 1996), p. 169.

${ }^{14}$ D. R. Penn, Phys. Rev. 128, 2093 (1962).

${ }^{15}$ A. C. Ferrari, A. Libassi, B. K. Tanner, V. Stolojan, J. Yuan, L. M. Brown, S. E. Rodil, B. Kleinsorge, and J. Robertson, Phys. Rev. B 62, 11089 (2002).

${ }^{16}$ J. Titantah and D. Lamoen, Phys. Rev. B 70, 033101 (2004).

${ }^{17}$ S. Bhattacharyya, S. J. Henley, E. Mendoza, L. Gomez-Rojas, J. Allam, and S. R. P. Silva, Nat. Mater. 5, 19 (2006). 